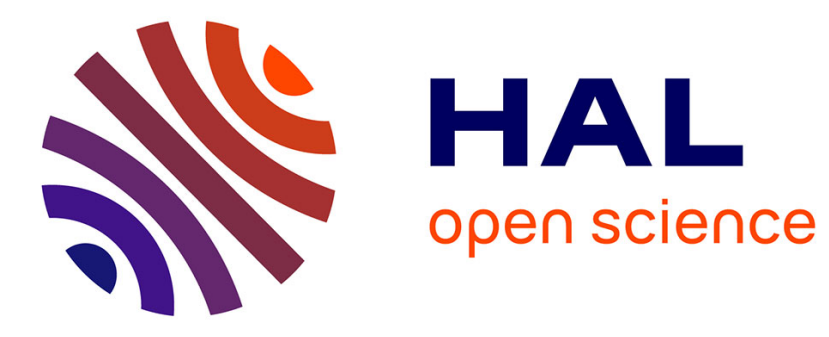

\title{
Du nouveau sur les comtesses catalanes (IXe-XIIe siècles) \\ Martin Aurell
}

\section{To cite this version:}

Martin Aurell. Du nouveau sur les comtesses catalanes (IXe-XIIe siècles). Annales du Midi: revue archéologique, historique et philologique de la France méridionale, 1997, pp.357-380. 10.3406/anami.1997.2564 . halshs-01306068

\section{HAL Id: halshs-01306068 https://shs.hal.science/halshs-01306068}

Submitted on 22 Apr 2016

HAL is a multi-disciplinary open access archive for the deposit and dissemination of scientific research documents, whether they are published or not. The documents may come from teaching and research institutions in France or abroad, or from public or private research centers.
L'archive ouverte pluridisciplinaire HAL, est destinée au dépôt et à la diffusion de documents scientifiques de niveau recherche, publiés ou non, émanant des établissements d'enseignement et de recherche français ou étrangers, des laboratoires publics ou privés. 


\section{Du nouveau sur les comtesses catalanes (IXe-XIle siècles)}

Martin Aurell

\section{Citer ce document / Cite this document :}

Aurell Martin. Du nouveau sur les comtesses catalanes (IXe-XIle siècles). In: Annales du Midi : revue archéologique, historique et philologique de la France méridionale, Tome 109, №219-220, 1997. pp. 357-380;

doi : 10.3406/anami.1997.2564

http://www.persee.fr/doc/anami_0003-4398_1997_num_109_219_2564

Document généré le 14/03/2016 


\title{
Zusammenfassung
}

Neues über die katalanischen Gräfinnen (9.-12. Jahrhundert).

Neuere Untersuchungen des Verfassers lassen die Herkunft von zweiundzwanzig katalanischen Gräfinnen des 9. bis 12. Jahrhunderts, die er selbst in zwei vorangegangenen Veröffentlichungen dargestellt hat, in neuem Licht erscheinen. Durch diese veränderte Sehweise wird das für die Grafen der KaroUngerzeit angenommene Schema einer Binnenheirat unter Blutsverwandten verfeinert. Diese ehelichen Verbindungen verwurzelten die Fursten zunehmend in Okzitanien, da sich in ihren Heiratsstrategien vor allem das Bemühen zeigt, ihre Beziehungen zu ihren nahen Verwandten aus dem Languedoc zu stärken ; der aus einer grenzüberschreitenden Eheverbindung herrührende Prestigegewinn war demgegenüber zweitrangig. Unter diesem Blickwinkel wird endlich auch die Eheschließung von Mahaut de Pouille, der Gattin von Raimund Berengar II., die bisher als eine Ausnahme von der okzitanischen Heiratsregel galt, auf der Grundlage einer neuen Quelleninterpretation verständlicher.

\section{Résumé}

Des travaux récents mettent en question ou complètent les données sur les origines de vingt-deux comtesses catalanes des IXe-XIle siècles, que l'auteur avait étudiées dans deux publications précédentes. Ces modifications nuancent le schéma de l'endogamie consanguine des comtes de la période carolingienne. Elles enracinent ces princes davantage en Occitanie : leurs stratégies matrimoniales visent bien plus l'efficacité de l'alliance avec leurs proches voisins languedociens que le prestige du mariage lointain. Enfin, les noces de Mahaut de Pouille, épouse de Raimond Bérenger II, l'une des exceptions à cette règle du mariage occitan, profitent désormais d'un éclairage documentaire plus net.

\begin{abstract}
New Information on the Catalan Countesses (9th-12th Centuries).

Recent works raise questions about or complete data on the origins of 22 Catalan countesses that the author had studied in two previous publications. These modifications bring nuances to the model of consanguineous endogamy that the counts were to have practiced during the Carolingean period. They root these princes more in Occitania : their matrimonial strategies were aimed more at establishing efficient alliances with their close Languedocian neighbors than at making prestigious far away ones. The wedding of Mahaut de Pouille, wife of Raymond Bérenger II, one of the exceptions to this rule of Occitanian marriages, can now, at last, be understood thanks to a clearer documentary background.
\end{abstract}




\section{Martin AURELL*}

\section{DU NOUVEAU SUR LES COMTESSES CATALANES (IX'-XII ${ }^{\mathrm{e}}$ SIËCLES)}

Dans la recherche médiévale, rares sont les exercices plus complexes que la reconstitution de la filiation d'une famille ou que l'identification des femmes qui s'y sont agrégées par mariage. Dans ce domaine, toute hypothèse de travail est aléatoire : elle résiste mal à la découverte de nouvelles sources, au perfectionnement d'une chronologie ou à l'affinement de la méthode onomastique. L'historien apprend la modestie dans ces études généalogiques, où la fréquence de ses rétractations ne répond pas tant à la légèreté, qu'à la rigueur d'une investigation en progrès. Il y découvre le travail en équipe. L'étendue de la géographie matrimoniale des lignées princières d'Occident dépasse, en effet, la connaissance habituelle des chroniques et des cartulaires de la seule principauté à l'intérieur de laquelle la micro-histoire régionale nous contraint souvent de mener une recherche ; elle exige de faire appel à d'autres spécialistes.

Par leurs remarques amicales, Claudie Amado, Hélène Débax, Pierre Bonnassie, Christian Settipani et Thierry Stasser ont corrigé quelques-unes des identifications des comtesses catalanes que nous avions proposées dans deux publications récentes ${ }^{1}$. Nous communiquons ici leurs découvertes, en les remerciants pour la générosité avec laquelle ils les ont mises à notre dis-

* 5, rue Dufrénoy, 75116 Paris.

1. «Jalons pour une enquête sur les stratégies matrimoniales des comtes catalans (IX c $^{\text {- }}$ $\mathrm{XI}^{\mathrm{e}}$ s.) », Symposium internacional sobre els origens de Catalunya (segles VIII-XI), Barcelone, 1991, t. I, p. 281-364 : les actes de ce colloque, indispensables pour l'histoire de la Catalogne médiévale, mais introuvables dans la plupart des bibliothèques françaises, sont diffusés par la Reial Acadèmia de Bones Lletres de Barcelona, C/Bisbe Caçador-8, E-08002 Barcelona. Les noces du comte. Mariage et pouvoir en Catalogne (785-1213), Paris, Publications de la Sorbonne, 1995, 623 p.

Professeur d'histoire médiévale à l'Université de Poitiers, Martin Aurell est l'auteur de nombreux ouvrages et articles sur l'histoire sociale des IX $^{\mathrm{e}}-\mathrm{XIII}{ }^{\mathrm{e}}$ siècles, principalement en Provence et en Catalogne. Parmi ses dernières publications, on retiendra Les noces du comte. Mariage et pouvoir en Catalogne (785-1213), Paris, 1995, et La noblesse en Occident ( $V^{e}-X V^{e}$ siècle), Paris, 1996. 
position. Leurs travaux et la parution d'études d'autres auteurs qui s'intéressent aux dynasties princières et à l'aristocratie de la Méditerranée occidentale justifient le présent article, où quelque vingt femmes des familles comtales de la Catalogne médiévale sont revisitées.

\section{Garsenda (d'Empúries et Roussillon), femme de Guifred II Borrell († 911), comte de Barcelone}

P. Bonnassie nous a rappelé l'hypothèse de ses origines toulousaines ${ }^{2}$. Ce point de vue avait, en effet, été soutenu par S. de Vajay, dans un article paru en $1980^{3}$ : sous sa plume, Garsenda devenait la fille du comte Eudes de Toulouse (879-918) et de Garsenda d'Albi (866) 4 . Cette identification ne semble cependant pas devoir être retenue. Elle se fondait, d'abord, sur le nom de Garsenda que notre comtesse partage avec l'épouse d'Eudes, sa prétendue mère. Mais $S$. de Vajay tirait, ensuite, un autre argument de la provenance albigeoise du nom de Riquilda, donné par Guifred II Borrel et sa femme Garsenda à leur fille ${ }^{5}$. Or, nous savons que ce nom existe bel et bien dans la maison de Barcelone au moment de la naissance de Riquilda : il est porté par la sœur de Guifred II Borrell lui-même ${ }^{6}$. La thèse toulousaine perd, en conséquence, du poids.

En revanche, l'idée de F. Valls, reprise par $R$. d'Abadal, faisant de Garsenda une fille de la maison d'Empúries-Roussillon paraît plus solide?. Elle suit l'histoire de la propriété du domaine de Tordera, situé dans le Maresme, dans le comté de Barcelone, dont la possession est confirmé en 862 à Sunyer Ier d'Empúries-Roussillon par Charles le Chauve. Sunyer II ( $†$ 915) en hérite de son père et le vend au juge Trasovad, qui le retrocède, à son tour, pour 2000 sous à Guifred II Borrell et à son épouse Garsenda en novembre $908^{8}$. Toutefois, en 986 , Tordera apparaît dans la

2. Leur mention est omise dans la notice de « Jalons... » (8), p. 307-308.

3. «Comtesses d'origine occitane dans la Marche d'Espagne aux $10^{\mathrm{c}}$ et $11^{\mathrm{e}}$ siècles », Hidalguia, 1980, p. 601-603.

4. Sur Garsenda d'Albi, cf. H. DÉBAX, «Les comtesses de Toulouse : notices biographiques », Annales du Midi, 1988, (6), pp. 218-219.

5. « Le nom de Richilde (Riquilda) n'étant pas utilisé auparavant à Barcelone, cette provenance doit être recherché du côté maternel (c'est-à-dire de Garsenda) », ibid., p. 601.

6. « Jalons... », (15), p. 313. Cf. C. SETTIPANI, Encore sur la succession et les alliances des comtes de Toulouse. Etudes sur quelques familles aristocratiques du Languedoc du IXe au $X I^{*}$ siècle, livre à paraître : le passage relatif à Garsenda se trouve, dans l'état actuel du manuscrit que l'auteur a eu l'extrême obligeance de nous remettre, autour de la note 84 .

7. Longuement développée par R. d'ABADAL, Catalunya carolíngia. Els diplomes carolingis a Catalunya, Barcelone, 1950-1952, p. 239-240.

8. Ego Trasovadus, vinditor vobis, domno Wifredo, comite hac marchio, que vocant Borrello, et uxori tue Gersinde, emptores... L'acte mentionne ensuite l'héritage au même endroit de Suniarius, comes, et uxor sua Ermingardim, J. RIUs, Cartulario de Sant Cugat del Vallès, Barcelone, 1945-47 n 3 (18 XI 908). Sunyer et Ermengarde pourraient être les parents de Garsenda, ce qui justifie leur citation dans la charte. 
liste des biens que Lothaire confirme à Sant Cugat ; or, ce document spécifie que Gausfred ( $†$ 991) d'Empúries-Roussillon en a fait donation à ce monastère pour accomplir une disposition testamentaire de son frère, le clerc Sunyer. Il en ressort que Tordera, ancienne possession des EmpúriesRoussillon, est retournée à cette famille à la mort de Guifred II Borrell, probablement par un don de sa veuve Garsenda, qui n'a eu aucun garçon, et qui cède aux siens cette villa qui leur appartenait naguère. Nous convenons que le raisonnement de $F$. Valls et $R$. $d$ 'Abadal est un peu trop subtil et soumis à trop de conjectures. Mais, en l'absence de documents, il semble plus convaincant que la seule homonymie entre l'épouse de Guifred II Borrell et Garsenda d'Albi, comtesse de Toulouse.

Nous gardons donc, avec la prudence de rigueur, la thèse de l'appartenance de Garsenda, fille probable de Sunyer II et Ermengarde, à la maison d'Empúries-Roussillon.

\section{Guinedilda (de Barcelone), femme de Raimond II ( $\uparrow 924)$, comte de Toulouse}

Une comtesse Guinedilda souscrit un acte dressé à Narbonne le 28 novembre $926^{9}$. Il ne peut s'agir de l'épouse de Guifred Ier $(† 897)$, comte de Barcelone, car elle est morte avant $904^{10}$. Elle est donc probablement la femme d'un comte de Toulouse ${ }^{11}$, sans doute Raimond II († 924), car elle appose son seing seule, en l'absence de son mari qu'on peut supposer décédé ${ }^{12}$.

Le nom de Guinedilda, extrêmement rare, est entré, avec le mariage de Guifred I I $^{\mathrm{e}}$ † 897), dans la maison de Barcelone, où il faut sans doute trouver les origines de la femme de Raimond II de Toulouse. C. Settipani propose d'en faire la fille de Guifred II Borrell et de Garsenda (d'Empúries et Roussillon), c'est-à-dire la sœur aînée de Riquilda, vicomtesse de Narbonne, qui souscrit la charte de 926 tout de suite après elle ${ }^{13}$. Son raisonnement emporte l'adhésion.

9. S. Guifridus, S. Tedmares, S. Walcarius, S. Widinildis comitissa, S. Richildis vicecomitissa, C. Devic, J. Vaissette, Histoire générale de Languedoc, Toulouse, 1875, t. V, n 52 , col. 151-152.

10. « Jalons », (4), p. 306. Cf. contra DEBAX, « Les comtesses... », (7), p. 219.

11. « La comtesse Guinilde de la charte de 926 à Narbonne était vraisemblablement un membre de la famille raymondine, qui possédait Narbonne. Elle peut être l'épouse de Raymond II, ou encore une première épouse non autrement attestée de Raymond Pons : on est réduit à des conjectures », T. STASSER, « Autour de Roger le Vieux : les alliances matrimoniales des comtes de Carcassonne », Annales du Midi, 1996, p. 173, n. 43.

12. SetTIPANi, Encore..., autour n. 110.

13. Ibid. Plutôt qu'une fille de Guifred $I^{\text {er }}(\dagger 897)$ et de Guinedilda comme le propose S. de Vajay, «Comtesses... », p. 604, n. 36. 


\section{Guinedilda (de Barcelone), femme de Raimond (961), comte de Toulouse}

Dans son testament, dicté autour de 972, la comtesse Garsenda, veuve de Raimond III Pons de Toulouse († 940/944), donne quelques alleux à son nepos Raimond, fils de Guinedilda ${ }^{14}$. H. Débax propose de faire ce cette Guinedilda la femme de Raimond II $^{15}$, mais on voit mal comment Garsenda pourrait traiter le frère de son mari de « neveu » ou de «petit-fils ». $M$. de Framond suggère plutôt de marier Guinedilda au comte Raimond, attesté en 961, fils de Raimond de Rouergue, cousin germain de Raimond III Pons ; cette filiation rendrait un peu mieux compte de l'utilisation du terme nepos par Garsenda, en l'occurrence pour désigner le petit-fils du cousin germain de son mari ${ }^{16}$. Nepos peut, en effet, revêtir un sens large, désignant les collatéraux d'une ou deux générations postérieures à l'intéressé.

Nous proposons d'identifier Guinedilda avec une fille de Miró II († 927), comte de Cerdagne-Besalú, qui la cite, le 13 juin 925, dans son testament avec les autres filles qu'il a eues d'Ava ${ }^{17}$. Elle est sans doute encore célibataire, voire mineure, à cette date. La chronologie n'empêche donc pas de la voir apparaître vers 961 en tant qu'épouse de Raimond de Toulouse.

\section{Adelaida de Barcelona, femme de Sunifred II († 940), comte d'Urgell}

C. Settipani et T. Stasser font remarquer le problème chronologique que pose ce mariage, mentionné pour la première fois, de façon trop précoce, dans un acte de novembre 90718. Or, I'on sait qu'Adelaida est la fille de Sunyer Ier de Barcelone et de Riquilda (de Rouergue) ${ }^{19}$, qui apparaissent pour la première fois mariés ensemble dans un document daté de $925^{20}$. L'acte de novembre 907, exclusivement connu par une transcription du XIII ${ }^{e}$ siècle, a sans doute été mal daté par son copiste ${ }^{21}$. Il faut attendre,

14. Cotnag et Valedias dono Raymundo, filio Gundinildis, nepoti meo. Post mortem ejus remaneat Santi Justi et Sancti Pastoris et Sancto Paulo Narbona et Sancto Nazario Biterris, Devic, VaisseTte, Histoire..., t. V, n 125, col. 278.

15. « Les comtesses... » (7), p. 219.

16. M. de Framond, « La succession des comtes de Toulouse autour de l'an mil (9401030) : reconsidérations », Annales du Midi, 1993, p. 488.

17. ad filia mea Guilindo facite scripturam de alode meo quod abeo in comitatu Ausona quod dicunt Devesa, vel hoc quod habeo in Mocones vel in ejus termine, P. de Bofarul., Los condes de Barcelona vindicados, Barcelone, 1836, t. 1, pp. 88-90 (13 VI 925).

18. Encore..., n. 95, et "Origine familiale de trois comtesses de Pallars », à paraître dans Anuario de Estudios Medievales, n. 25.

19. Ego Adalaiz, comitissa, que vocant Bonafilia, filiam Suniarium, comitem, et Richildis, chomitissam, F. UdinA, El archivo condal de Barcelona en los siglos IX-X, Barcelone, 1951, $n^{\circ} 130$ (2 VI 950).

20. «Jalons... », (12), p. 311.

21. C. BARAuT, « Els documents dels segles IX i X conservats a l'Arxiu Capitular de la Seu d'Urgell », Urgellia, 1979, p. 54-55, nº 65 (XI 907). 
ensuite, vingt-huit ans pour voir, en 935, à nouveau Adelaida avec Sunifred II $^{22}$. En somme, leurs noces ont dû se célébrer autour de cette dernière date, plutôt qu'avant 907 .

\section{Aimilda (de Toulouse) et Riquilda (de Rouergue), femmes de Sunyer Ier († 950), comte Barcelone}

Les études récentes de C. Settipani et de T. Stasser nous ont aidé à comprendre que nous avions rejeté de façon injuste l'identification, proposée par S. de Vajay en 1980 , de Riquilda, épouse de Sunyer Irer, avec une Rouergate $^{23}$. L'argumentation de S. de Vajay ne se fonde pas seulement sur le nom de cette femme, mais surtout de deux de ses enfants - Ermengol et Adelaida - qui viennent de la maison comtale de Toulouse-Rouergue ${ }^{24}$. Il existe donc de fortes chances pour que les parents de Riquilda soient Ermengol de Rouergue ( $†$ 936/944) et Adelaida (de Bourgogne) ${ }^{25}$.

Les Archives de la Couronne d'Aragon conservent l'original d'un acte, dressé sous le règne de Charles le Simple (898-922), aux termes duquel Sunyer I $^{\text {er fixe le douaire de sa femme Aimilda }}{ }^{26}$. Nous avions pensé que le scribe avait donné ce nom pour celui de Riquilda ${ }^{27}$. Une lecture de la charte originale nous a, pourtant, montré que le nom d'Aimilda s'y lit très clairement. En conséquence, même si Aimilda n'apparaît que dans ce seul document, il n'y a pas de raison majeure pour nier son existence ${ }^{28}$. Cette femme pourrait bien être fille de Raimon II de Toulouse ( $†$ 924) et d'Aimilda (d'Auvergne) ${ }^{29}$.

22. Ibid. $\mathrm{n}^{\circ} 96(21$ VII 935$)$.

23. «Jalons...», (12), p. 312.

24. «Comtesses... ", p. 598.

25. "L'hypothèse de $S$. de Vajay prend une force non négligeable ", SETripani, Encore..., autour n. 93-95. «S. de Vajay a montré par des nombreux arguments que Richilde, seconde épouse du comte de Barcelone Suniaire, était sans doute une fille du comte de Rouergue », STASSER, «Autour... », p. 174.

26. UdiNA, El archivo condal..., n ${ }^{\circ} 9$, p. 116-118.

27. « Jalons», (12) p. 311.

28. «M. Aurell croit ici à une erreur textuelle et confond Emilde avec Richilde, seconde épouse de Suniaire, solution drastique que rien ne justifie », SETTIPANI, Encore..., n. 119.

29. Ibid. Cf. VAJAY, «Comtesses... », p. 763-764, où Aimilda provient de la maison de Gévaudan. 


\section{Ledgarda et Aimeruda (d'Auvergne), femmes de Borrell II († 992), comte de Barcelone}

D'après C. Settipani, la chronologie empêcherait de considérer sœurs Ledgarda et Aimeruda, femmes successives de Borrell II $^{30}$. Nous ne le pensons pas. Borrell II épouse Ledgarda vers 967 et Aimeruda peu avant 988 , après son veuvage. Ce laps d'une vingtaine d'années dans le mariage de deux sœurs à Borrell II n'a rien d'extraordinaire, si l'on considère que Ledgarda est l'aînée d'Aimeruda devenue, d'un lit précédent, la mère $d^{\prime}$ 'Aldria ${ }^{31}$. Aimeruda est ainsi veuve au moment où elle devient la seconde femme de Borrell II. Il n'empêche que C. Settipani remarque, à juste titre, que l'hypothèse ancienne d'une filiation commune de ces deux dames, avancée par E. Baluze et reprise par P. de Bofarull ${ }^{32}$, n'est fondée sur aucune source précise.

\section{Teutberge (de Forez et Lyonnais), femme d'Ermengol Ier († 1010), comte d'Urgell}

Le nom de cette comtesse est très rare dans le Midi, ce qui facilite une recherche onomastique parmi les familles princières. S. de Vajay proposait d'en faire la fille de Roubaud II de Provence ( $\dagger$ 1008), dont l'arrièregrand-mère pourrait être Teutberge de Troyes $^{33}$. A. de Fluvià nous avait conseillé une filiation se fondant sur une continuité onomastique plus rapprochée, pour voir en elle une fille de Pons Ier de Gevaudan et de Teutberge (de Maurienne ou de Limoges) ${ }^{34}$. T. Stasser vient de montrer que cette théorie n'est pas recevable. En fait, Teutberge (de Maurienne ou de Limoges) n'épouse Pons Ir qu'en secondes noces, après son veuvage ; elle est auparavant la femme d'Artaud, comte de Forez et Lyonnais, jusqu'à l'an mil, année de la mort de ce dernier. Or, le mariage de sa fille homonyme et d'Ermengol Ier intervient également autour de l'an mil ; celle-ci ne peut, par conséquent, qu'être née du premier lit de sa mère. En définitive, si elle est la fille de Teutberge (de Maurienne ou de Limoges), il y a de fortes chances que son père soit Artaud de Forez ${ }^{35}$.

30. SETTIPANI, Ibid., n. 115.

31. « Jalons...», (19) et (20), p. 314-316.

32. E. Baluze dans P. de MARCA, Marca hispanica sive limes hispanicus, Paris, 1688 , col. 401-402, cité par P. de Bofarull, Los condes..., t. I, p. 153.

33. « Comtesses... », p. 759-760.

34. « Jalons...», (23), p. 319.

35. T. STASSER, « Adélaïde d'Anjou. Sa famille, ses mariages, sa descendance », à paraître dans Le Moyen Âge, n. 90. 


\section{Mahaut de Pouille, femme de Raimond Bérenger II $(\dagger$ 1082), comte de Barcelone}

Deux ouvrages récents en langue française relatifs aux Normands en Italie méridionale mentionnent ce mariage ${ }^{36}$, attirant 1 'attention sur des sources inconnues des historiens de la Catalogne ${ }^{37}$. Voici ces trois nouveaux documents :

- La geste de Robert Guiscard (fin $\mathrm{XI}^{\mathrm{e}}$ siècle), rédigée par Guillaume de Pouille, clerc de l'entourage du duc : "Le noble comte Raimond de Barcelone était venu d'Hespérie dans cette ville (Salerne) afin de demander en mariage une fille du duc. On lui donna l'aînée. ${ }^{38}$ "

- L'histoire de l'invention des reliques de saint Sabin par Jean, archidiacre de Bari : " (Robert Guiscard) avait souvent envoyé (Ourson, archevêque de Bari), auprès du pape. Il le députa en Espagne quand il donna en mariage sa fille au comte de Barcelone. (Ourson) devait conduire ce comte, avec un entourage très fourni et en grand apparat, depuis le royaume de sa province jusqu'à la terre qui se trouvait sous la domination du duc, afin de confirmer les noces. ${ }^{39}$ »

- L'Alexiade (vers 1140) par Anne Comnène, fille de l'empereur Alexis : "Quant aux deux autres (filles), il maria l'une à Raimond, fils du comte de Barcelone ; il unit la seconde à Ebles (de Roucy), qui était lui aussi un comte très illustre. Toutes ces alliances ne semblaient pas du tout inutiles à Robert, car ainsi de tous côtés se constituait et s'augmentait à son profit une puissance, résultant de la naissance, du pouvoir, de la parenté et de mille autres causes auxquelles personne n'aurait songé ${ }^{40} »$.

36. J.-M. MARTIN, Italies normandes, XIe-XIIe siècles, Paris, 1994, p. 41, et H. TAVIANICAROzZI, La terreur du monde. Robert Guiscard et la conquête normande en Italie, Paris, 1996, p. 510.

37. Elles avaient également échapé à la sagacité de S. de Vajay dans son article « Mahaut de Pouille, comtesse de Barcelone et vicomtesse de Narbonne dans le contexte social de son temps ", Béziers et le Biterrois. 43e Congrès de la Fédération historique de LanguedocRoussillon, Montpellier, 1970, pp. 129-150, qui nie, de façon inexacte, à la suite de $\mathrm{S}$. Sobrequès, les fondements documentaires du voyage de l'archevêque de Bari à Barcelone, ibid. p. 138, n. 32.

38. Partibus Esperice, que Barcilona tenebat, venerat insignis comes hanc Raimondus ad urbem, ut nuptura ducis detur sibi filia poscens. Huic maior natu numbtum datur, éd. et trad. ci-dessus par M. MATHIEU, Palerme, 1961, p. 204-205.

39. Nam et frequenter ad predictum papam legaverat. Et in Hispaniam illum miserat, quando filiam suam comiti Barzellonensi nuptui dederat. Quem de suo privinciœ regno pro numtiis confirmandis usque in terram istam, quoe sub eius ducis erat dominio, cum multo comitatu et apparatu conduxerat, Historia inventionis s. Sabini, dans Acta Sanctorum, Bruxelles-Anvers, 1643-1940, 9 février, p. 329.

40. Éd. grecque et traduction par B. LEIB, Paris, 1937, t. I, p. 46-47. D'après M. Mathieu (dans son édition de Gurllaume de Poullle, La geste..., p. 45, n. 3), Anne Comnène a consulté les Gesta comitum Barchinonensium, mais elle écrit vers 1140 et les Gesta comitum ne sont mis en forme qu'autour de 1180. 
Enfin, P. Bonnassie vient d'attirer notre attention sur le testament d'un certain Gaufred Guillem qui fait l'objet d'une publication judiciaire à Gérone le 4 novembre 1080 et qui mentionne un voyage mené quelques mois auparavant par l'évêque de cette ville en Pouille, probablement pour négocier ce mariage. Gaufred Guillem a en effet dicté ses dernières volontés « en revenant de Pouille avec son seigneur Bérenger, évêque de Gérone, en haute mer, sur la nef que l'on appelle "Chat" en langue vulgaire, alité en raison de la maladie dont il devait mourir $\gg^{41}$.

Le second de ses témoignages nous semble le plus intéressant. Il mentionne notamment un voyage de Raimond Bérenger II en Italie. Il insiste, par ailleurs, sur le fait qu'Ourson, élu archevêque de Bari à partir d'août 1080 , a mené auparavant le cortège nuptial de la fille de Robert Guiscard à Barcelone. Cet ecclésiastique est un habitué des ambassades au Latran, où il a dû traiter de la levée de l'excommunication qui pèse sur son maître entre 1074 et juin 1080, année où Grégoire VII s'entend avec lui pour lutter contre Henri IV ${ }^{42}$.

Les sources barcelonaises révèlent que le mariage de Mahaut intervient peu avant juillet 1078 , tandis que son père, contre lequel le pape vient de renouveler l'interdit, combat les troupes pontificales à Bénévent. Pourtant, les historiens ont l'habitude de voir, derrière cette union matrimoniale, la main de Grégoire VII qui s'appuya sur Ebles de Roucy, autre gendre de Robert Guiscard, pour faire progresser la Reconquista ; ils mettent l'accent sur l'alliance ultérieure entre la papauté et les Normands d'Italie contre l'empereur romano-germanique ${ }^{43}$. Il n'empêche que la chronologie du mariage de Mahaut ne laisse aucune part à l'initiative du pape, qui était à l'époque des noces l'ennemi déclaré de son père. Ce n'est qu'à partir de l'été 1080 que le renversement des alliances aurait permis à Grégoire VII de tirer profit des liens matrimoniaux tissés naguère par Robert Guiscard. Ourson de Bari a dû jouer alors son rôle de médiateur.

\section{Ermengarde (de Poitiers), femme de Sunyer II $(\dagger 915)$, comte d'Empúries et Roussillon}

Trois hypothèses ont été récemment avancées sur les origines de cette dame ${ }^{44}$.

41. Rediens a partibus Apulie cum dompno Berengario Gerundensi episcopo, seniore suo, jacens in ipsa navi superposita mari qui rustico nomine dicitur Catus, detentus ab egretudine qua obiit, J.-M. MARQues, Cartoral, dit de Carlemany, del bisbe de Girona (s. IX-XIV), Barcelona, 1993, t. I, n 148.

42. Mathieu, dans Guillaume de Pouille, La geste.... p. 205.

43. « Jalons... » (36), p. 330-331 et Noces, p. 58.

44. Filiation inconnue dans "Jalons", (41), p. 334, où une faute d'impression donne une mauvaise date pour la plaque commémorative de 927. 
1) Le nom de son fils Benció est celui d'un comte de Carcassonne, peutêtre le frère d'Ermengarde ${ }^{45}$.

2) Le nom de son fils Gausbert et de son petit-fils Gausfred sont caractéristiques des Roriconides du Maine. Or, autour de 860 , Bilichilde, une fille de cette famille, épouse Bernard, comte de Poitiers. Ermengarde aurait pu être née de cette union, qui en ferait, du côté de sa mère, la nièce d'un Gausbert et la petite-nièce d'un Gausfred ${ }^{46}$.

3) Blichilde épouse, en secondes noces, Ramnulf I $^{\mathrm{er}}$, autre comte de Poitiers, dont le grand-père maternel, Pépin († 838), roi d'Aquitaine, était le fils de Louis le Pieux et d'Ermengarde. La naissance d'Ermengarde, femme de Sunyer II, de Ramnulf Ier de Poitiers el de Blichilde du Maine, semble ainsi la plus vraisemblable des hypothèses ${ }^{47}$.

\section{Trudegarde de Narbonne, femme de Gausbert Ier $(\$ 931)$, comte d'Empúries et Roussillon}

T. Stasser voit en elle la fille de Francon ( $\dagger 924)$, vicomte de Narbonne, et d'Arsinda de Toulouse. Les synchronismes ne s'y opposent pas. Le nom Trudegarde est porté par l'une de ses petites-nièces dans la maison de Narbonne. Toutefois, cette filiation expliquerait surtout pourquoi, en 931, Gausbert Ier offre, dans le même diplôme, des suffrages pour Sunyer II et Ermengarde, ses parents, mais aussi pour Francon, Arsinda et Eudes, vicomtes de Narbonne, devenus respectivement, grâce à cette conjoncture, ses beaux-parents et son beau-frère ${ }^{48}$. Cette argumentation nous semble difficile à réfuter ${ }^{49}$. Elle se fonde sur l'évidence documentaire.

Elle rend peu probable l'hypothèse de C. Amado, d'après laquelle Trudegarde provient de la famille de seigneurs de Saint-Amans et de Teulet, ancêtres des Guilhem de Montpellier, En faveur de cette théorie, cette médiéviste avance tout un faisceau de coïncidences reliant les SaintAmand-Teulet, les Guilhem et les comtes de Roussillon, qui explique

45. T. STASSER, « La maison vicomtale de Narbonne aux $\mathrm{X}^{*}$ et $\mathrm{XI}^{\mathrm{E}}$ siècles », Annales du Midi, 1993, p. 499. N. 15.

46. P. Ponsich, « À propos des attaches familiales de Girart de Roussillon en Catalogne », La chanson de geste et l'épopée carolingienne, Paris, 1982, II, p. 770.

47. Elle est la plus sûre d'après C. Settipani, Encơrè..., n. 234, et sa communication encore inédite au congrès de Leeds de juillet 1996.

48. Stasser, «La maison vicomtale... », p. 495, et Devic, Vaissetre, Histoire..., t. V, $\mathrm{n}^{\circ} 55$, II, col. 157 ( 10 IV 931 ).

49. C. Amado s'y oppose, cependant. Elle nous écrit dans sa lettre du 7 septembre 1996 : «La démarche de Stasser semble tracer une équation simple : suffrages de Gausfred (I) en faveur de ses beaux-parents et de son beau-frère. Après tout, les liens qui unissent, depuis des décennies et pour des décennies, les lignées comtales catalanes et la famille de Narbonne, au temps de Gausbert, recèlent sans doute l'explication sans avoir à recourir à Trudegarda ». 
l'adoption du prénom de Trudegarde par deux membres de la famille de Saint-Amans et Teulet ${ }^{50}$.

\section{Ava (de Cerdagne ou de Ribagorça), première femme de Gausfred Ier († 991), comte d'Empúries et Roussillon}

A. de Fluvià la considère fille de Miró II de Cerdagne († 927) et d'Ava, fille de Fidel ${ }^{51}$. T. Stasser se demande plutôt si elle ne serait pas la fille de Bernat Unifred ( $†$ 950/956), comte de Ribagorça, femme mentionnée par les Généalogies de Roda à la fin du $\mathrm{X}^{\mathrm{c}}$ siècles2. Les données chronologiques ne s'opposent pas à cette hypothèse : Ava n'est, en effet, citée qu'en 971 en tant que mère de Sunyer d'Empúries et Roussillon, évêque d'Elne.

\section{Guisla (de Melgueil), seconde femme de Gausfred Ier $(† 991)$, comte d'Empúries et Roussillon ${ }^{53}$}

S. de Vajay tient cette dame pour une fille de Raimond de Rouergue et de Berthe de Toscane, fille à son tour d'une Willa, dont il assimile le nom à celui de Guisla. Cette filiation présente l'avantage de rendre compte des noms de trois des enfants de Gausfred Ier d'Empúries et de Roussillon et de Guisla, à savoir Hug, Arsinda et Sengunda portés dans la maison de Rouergue ${ }^{54}$.

Cette hypothèse vient d'être corrigée par $C$. Settipani qui fait naître Guisla de Bernard II, comte de Malgueil, précisément le fils d'une Guisla, et de Sengunda de Rouergue, dont on retrouve de même le nom chez une fille du comte d'Empúries et Roussillon. C'est ainsi par leur grand-mère maternelle qu'Hug et Arsinda auraient reçu des noms rouergats ${ }^{55}$.

\section{Beliarde, femme de Guislabert Ier († 1013), comte de Roussillon}

C. Amado nous indique que le nom de Belliarde pourrait provenir des lignées languedociennes des Cévennes et de leurs piémonts, au regard des chartes de Notre-Dame de Nîmes et de Lodève ${ }^{56}$.

50. С. Am^do, La famille aristocratique languedocienne. Parenté et patrimoine dans les vicomtés de Béziers et d'Agde (900-1170), Thèse d'État en cours d'édition, Université de Paris IV, 1994, t. II, p. 267-271.

51. Els primitius.., p. 114, repris par « Jalons... », (45), p. 335.

52. Lettre du 4 décembre 1996. R. d'Abadal avait identifié, à tort, cette Ava à l'épouse de Miró II de Cerdagne, « Jalons... », (9), p. 309.

53. « Jalons... », (46), p. 335-336.

54. VAJAY, «Comtesses... », p. 768.

55. SetTIPANi, Encore..., autour n. 229.

56. Dans sa lettre datée du 7 septembre 1996. 


\section{Trudegarde (d'Empúries et Roussillon), mère de Guilhem II Bernard $(\dagger$ 1043), seigneur de Montpellier}

Cette dame est citée en 989 dans le testament de son père Gausfred I ${ }^{\text {er }}(† 991)$, comte d'Empúries et Roussillon ; la teneur de ce document laisse supposer qu'elle est encore célibataire ${ }^{57}$. Elle pourrait bien être la mère de Guilhem II Bernard, seigneur de Montpellier, cité comme fils d'une Trudegarde qui l'accompagne dans un acte de Gellone, daté de 1019-1022 par C. Amado ${ }^{58}$. Trudegarde ne serait donc pas la fille de Gui (985), fondateur de Montpellier, comme le propose cette historienne, mais sa bru. Il est, en effet, plus vraisemblable que Guilhem II Bernard ait reçu sa seigneurie par son père plutôt que par sa mère. Ce sont donc les comtes d'Empúries et Roussillon qui auraient introduit, au début du $X^{\text {e }}$ siècle, le nom de Trudegarde dans la famille des Guilhem. Si la théorie de T. Stasser était exacte ${ }^{59}$, les Empúries-Roussillon auraient, à leur tour, repris ce nom aux Narbonne au début du $\mathrm{X}^{\mathrm{e}}$ siècle.

Il faut cependant rappeler les trois arguments utilisés par C. Amado afin de faire de Trudegarde la fille de Gui (985), car ils ne manquent pas d'intérêt.

1) Guilhem Ier de Montpellier ( $\dagger$ 1022), Bérenger et Pierre sont bien attestés en tant que frères. D'ailleurs, peu avant 1022, Guilhem I ${ }^{\text {er }}$ apparaît dans un document avec son neveu Guilhem II Bernard, son épouse Béliarde, et une Trudegarde que doit être probablement la mère de Guilhem II Bernard, sœur de Guilhem Ier.

2) On ne connaît pas le nom du père de Guilhem II Bernard, qui hérite de la grande seigneurie que celui-ci aurait constituée. Si ce personnage inconnu était le fils de Gui et d'Ingilrada, une Nîmoise, nous l'aurions vu dans l'acte impliquant Ingilrada, déjà veuve, et ses trois autres fils, à savoir Guilhem Ier, Bérenger et Pierre.

3) Il est de tradition dans la famille des Guilhem de rédiger très soigneusement les clauses substitutives dans les testaments des hommes de la famille, du moins à partir du $\mathrm{XI}^{\mathrm{e}}$ siècle. L'ordre substitutif est alors le suivant : d'un frère mort intestat à un autre frère, et en l'absence d'un héritier mâle, aux mâles nés d'une sœur ; Trudegarde pourrait, en conséquence, avoir transmis à son fils Guilhem II Bernard son droit d'accéder à l'héritage par voie substitutive ${ }^{60}$.

57. Et ad filias meas his nominibus Trudgardis, Arsendis, Senegundes et a predicta Visla ipsos meos alodes quod abeo in comitatu Empuritano, M.I. Simo, « Aportación a la documentación condal catalana (siglo X) », Miscellanea de estudios dedicados al professor Antonio María Oceto, Grenade, 1974, n 11 (20 II 989). Cf. « Jalons... », (46) et (49), p. 335-336.

58. La famille..., t. II, p. 270 , n. 18.

59. Cf. ci-dessus p. 365.

60. Lettre du 7 septembre 1996. 


\section{Ermengarde (de Roussillon), femme d'Oliba Cabreta $(\uparrow 990)$, comte de Besalú et Cerdagne}

Elle détient l'alleu de Custujà, situé dans le pays d'Elne, dans le comté de Vallespir, qu'elle remet à son mari, et l'alleu de Llupià, sis au même endroit, dont elle fait donation à Saint-Martin du Canigou ${ }^{61}$. Il y a de fortes chances qu'elle ait hérité ces biens de ses parents. Or, le nom d'Ermengarde avait été introduit dans la famille de Roussillon par la femme de Sunyer II $(† 915)^{62}$. En conséquence, la double conjonction du patrimoine et de l'anthroponomic pousse à trouver, sans ambages, les origines de cette dame dans la maison comtale du Roussillon. A. de Fluvià en fait ainsi la fille probable de Gausbert I ${ }^{\text {er }}(† 931)$, comte d'Empúries, et Trudegarde ${ }^{63}$, tandis que T. Stasser la veut plutôt, une génération plus tard, la fille de Gausfred Ier $(\dagger 991)$ et d'Ava $^{64}$. La chronologie convient mieux à cette seconde filiation : les chartes citent, en effet, Ermengarde pour la première fois le 26 août 967, récemment mariée à Oliba Cabreta, et placent son décès autour de $1007^{64}$.

C. Settipani préfère voir chez Ermengarde la fille de Barnard (†980-9), comte de Melgueil ${ }^{60}$, en raison des noms de Bernard, de Bérenger et d'Adelaida, qu'elle introduit dans la maison de son mari. Le point faible de cette identification se trouve, cependant, dans l'existence des alleux d'Ermengarde dans le Vallespir, qu'on ne saurait pas faire remonter à ses aïeules. Il est rare, en effet, qu'une grand-mère puisse, à l'époque, transmettre des biens à sa petite-fille; le plus souvent, sa dot est récupérée par ses fils, qui les cèdent en douaire à leur femme ou qui les donnent à des établissements religieux.

\section{Goltregoda (de Barcelone), femme de Llop Ier $(†$ 947), comte de Pallars}

Traditionnellement, les historiens catalans - et en particulier R. d'Abadal - identifient cette dame à l'une des filles que Miró II $(† 925)$, comte de Besalú et Cerdagne, cite dans son testament ${ }^{67}$.

61. Ego Ermengards, comitissa, donator sum tibi viro meo Oliha, gratia Dei comes (...) de aliquo de proprietate mea (...) alaudem vocitatum nomine Custuja, qui est in pago Elenense in comitato Valle Asperi, MARCA, Marca hispanica..., n 138 (14 II 988) ; Ego Ermengardis. gratia Dei comitissa, donatrix sum domino Deo et Sancto Martino montis Kanigonis (...) alaudem meum proprium qui michi advenit per parentorum meorum vel per comparatione sive per qualicumque voce, B. AI.ART, Cartulaire roussillonnais, Perpignan, 1880, n 23 (1007).

62. Ci-dessus p. 364-36.5.

63. Els primitius comtats i vescomtats de Catalunya. Barcelone, 1989, p. 54.

64. «Origine...) (III), autour n. 34.

65. BorARULl, Los condes..., t. I p. 94 et «Jalons... », (60), p. 339-340.

66. Encore..., autour n. 235.

67. Ad filia mea Goltregoto facite scripturam in comitatu Cerdanie de alode meo que dicunt Villanova vel in ejus termine, BofARULL, Los condes..., t. I, pp. 88-90 (13 VI 925). Cf. ABADAL. Els comtats..., p. 133-136, « Jalons... », (64), p. 340-342, et ci-dessus p. $360 . C f$. contra STASSER, «Origine »... 
T. Stasser avance, toutefois, quatre arguments, convaincants pour la plupart, afin de la rendre fille de Sunyer ( $\uparrow 948)$, comte de Barcelone, et de Riquilda.

1) L'alleu de Villanova, légué par Miró II à sa fille Goltregoda, n'apparaît pas dans la documentation de la comtesse de Pallars. Nous écartons cette première critique dans la mesure où elle se fonde sur l'argument $a$ silentio de sources qui ne sont pas extrêmement abondantes.

2) En revanche, la possession de biens situés dans le comté de VicAusona, qui appartient au comte de Barcelone, par Goltregoda est un argument bien plus décisif.

3) Les noms des quatre derniers enfants de Goltregoda appartiennent à la maison de Barcelone. R. d'Abadal voyait dans ce choix une option politique pro-barcelonaise de Goltregoda de Cerdagne et Besalú, mais il est plus simple de l'expliquer par la règle de la filiation dans la transmission des noms au sein des familles aristocratiques.

4) Borrell II $(+992)$ de Barcelone appelle nepotes les fils de Goltregoda, sa sœur dans l'hypothèse de T. Stasser. Il seconde, d'ailleurs, cette dame, devenue veuve, dans le gouvernement du comté de Pallars.

\section{Tota-Adelaida (de Toulouse), femme de Bernard Tallaferro $(† 1020)$, comte de Besalú}

À la suite d'une hypothèse d'A. de Fluvià, nous avions considéré TotaAdelaida comme la fille d'Henri Ier, duc de Bourgogne, et de Garsenda de Gascogne ${ }^{68}$. Les prénoms d'Henri et d'Hugues qu'elle introduit dans la maison de Besalú apparaissaient ainsi Capétiens. C. Settipani a démonté ce point de vue ${ }^{69}$ : le mariage entre Henri Ier et Garsenda date de 986 ; or, en 994, Adelaida, leur prétendue fille, apparaît, à la suite de son mariage, installée dans le comté de Besalú, où elle rend justice. Elle ne saurait avoir guère plus que sept ans. En se fondant sur les noms de ses enfants, cet historien propose de voir plutôt en elle la fille de Guilhem II (†993/4), comte de Provence, et d'Arsinda de Carcassonne.

Cette hypothèse rend, pourtant, mal compte du mariage ultérieur entre Guillem Ier ( $\dagger$ 1052) de Besalú, fils de Bernard Tallaferro et de TotaAdelaida, et Adelaida de Provence, qui apparaît désormais comme sa tante maternelle. Pour parer à cette objection, C. Settipani critique l'interprétation traditionnelle de la lettre de 1020, par laquelle les moines de Ripoll annoncent la mort de Bernard Tallaferro, qui s'est noyé en traversant le Rhône alors qu'il cherchait une femme pour son fils ${ }^{70}$. Ce document ne dit

68. « Jalons... », (66), p. 341, et Les noces..., p. 55-56, et Fluvià, Els primitius..., p. 50.

69. Encore..., autour n. 189.

70. Talis igitur cum esset ac tantus ut pote provisor omnium, conjugali vinculo volens nectere filium, promisso jam nato, infando tentavit equo transire Rhodanum. MARCA, Marca.... ap. $\mathrm{n}^{\circ} 187$ (26 IX 1020), et « Jalons... », (72), p. 344-345. 
pas, en effet, qu'il ait voyagé en Provence, mais seulement au-delà du fleuve. Toutefois, cette indication topographique nous semble assez précise pour désigner la Basse Provence rhodanienne, avec ses grands comtés d'Arles et d' $\Lambda$ vignon. Unie à l'existence du prénom d'Adelaida dans cette maison, elle rend probable l'idée du mariage provençal de Guillem Irer.

Une nouvelle hypothèse proposée par T. Stasser mérite davantage d'être retenue $^{71}$. Elle trouve les parents de Tota-Adelaida en Raimond de Toulouse (972) et Adelaida d'Anjou, qui l'épouse en secondes noces, avant son mariage avec Louis V de France, puis avec Guilhem II de Provence. On explique de la sorte l'adoption des prénoms d'Hugues, Guilhem et Garsenda, présents dans la maison de Toulouse et Rouergue, par les enfants de Tota-Adelaida. Par ailleurs, Guilhem III Taillefer ( $\dagger$ 1037), frère ou oncle-paternel de Tota-Adelaida, appelle Henri l'un de ses enfants, nom que porte un fils de notre comtesse.

\section{Ermengarde (d'Empúries et Roussillon ou de Cerdagne), femme de Borrell I ${ }^{\text {er }}\left(\dagger\right.$ 995), comte de Pallars ${ }^{72}$}

T. Stasser suggère d'abandonner la piste de ses origines rouergates, chère à $\mathrm{S}$. de Vajay et $\mathrm{A}$. de Fluvià, dans la mesure où Goltregoda, qu'il sait désormais issue de la maison de Barcelone, aurait pu introduire el nom d'Ermengol chez les Pallars. Ce chercheur voit plutôt en elle une fille de Gausferd Ir († 991) d'Empúries et Roussillon et d'Ava ou bien d'Oliba Cabreta $(\dagger 990)$ de Cerdagne et d'Ermengarde : ces deux conjectures permettent d'expliquer le nom d'Ava qu'elle donne à l'une de ses filles ${ }^{73}$.

\section{Ermentrude, première femme de Sunyer Ier $(\dagger 1011)$, comte de Pallars}

T. Stasser a découvert le nom de cette dame, mère de Raimond IV de Pallars Jussà, dans un serment de fidélité qui, en dépit de sa publication,

71. « La maison vicomtale... », p. 497-498, n. 52, et « Adélaïde d'Anjou », (10), autour de la n. 153.

72. «Jalons... », (98), p. 357. En ce qui concerne les origines de la maison de Pallars, corriger Les noces..., tableau VI, p. 562 : Raimond de Pallars est, d'après une charte d'avril 920 , le fils de Loup, et non pas de Donat Loup (Regimundo, filio Luponi comiti (...) in pago Paliarensi, Abadal, Els comtats..., t. I, 2, n 132). Cf. SeTripani, Encore..., n. 47, et, avec un schéma légèrement différent sur les origines de cette maison, STASSER, « La maison... », p. 493, n. 32 .

73. «Origine... », (III), autour n. 27. 
avait été négligé par les généalogistes catalans ${ }^{74}$. Il est difficile de déceler ses origines familiales.

\section{Ermessende (d'Urgell), seconde femme de Raimond IV $(\dagger$ 1047), comte de Pallars Jussà}

Le même serment de fidélité qui nous apprend que Raimond IV était le fils d'Ermentrude livre le nom de la mère de sa seconde femme : Ermessende y apparaît en tant que fille de Gilga. Peut-être cette dernière femme était-elle Guisla, seconde épouse d'Ennengol Ior († 1007) d'Urgell, comme le propose T. Stasser ? La conjoncture politique est, en tout état de cause, favorable à cette union, alors que les maisons d'Urgell et de Pallars se rapprochent dans les années $1020^{75}$.

\section{Estefania (de Forez), femme de Guillem II $(\dagger$ 1035), comte de Pallars Sobirà}

A. de Fluvià proposait de voir une fille d'Ermengol I'r d'Urgell dans l'épouse de Guillem II de Pallars Sobiràa ${ }^{76}$, mais cette hypothèse ne saurait être retenue. Puisque la date de son mariage empêche de faire de Teutberge, prétendue femme d'Ermengol Ier et mère d'Estefania, une fille de Pons de Gévaudan († 1016/1018) et de Teutberge de Fore $z^{77}$, son prénom ne peut pas provenir directement de la maison de son grand-père maternel.

T. Stasser croit plutôt pouvoir faire naître Estefania du premier mariage de ce même Pons de Gévaudan avec une dame dont le nom est inconnu. S'il en était ainsi, le prénom d'Estefania (Étiennette) s'expliquerait aisément, car Pons de Gevaudan est le fils d'Étienne de Brioude et le père de l'évêque Étienne de Clermont. Un problème, mis en valeur par ce chercheur, subsiste cependant. Guillem II de Pallars Sobirà et Estefania appellent Artaud l'un de leurs fils. Or, Artaud est le prénom de l'assassin de Pons de Gévaudan, tué par son propre beau-fils, né d'une union précédente de sa seconde femme Teutberge avec Artaud de Forez. Comme ce meurtre intervient avant le mariage entre Guillem II et Estefania, il est difficile d'admettre que cette femme donne le nom de l'assassin de son propre père à l'un de ses fils.

74. Iuro ego Radulf Oriol, filium Mirabile, ad te Raimundo chomite, filium Ermetruete, et a te Ermessende, chomitisse, filia Gilga, de ipsos chellos de Aringo et de Oriti, F. MIQUEL, Liber Feudorum Maior, Barcelone, 1945, $\mathrm{n}^{\circ} 141$ : Ermessende est le nom de l'épouse de Raimond IV de Pallars Jussà ; Areny (Conca de Tremp) et Orrit (Robagorça) se trouvent dans ou à proximité du comté de Pallars. Cf. T. STASSER, « Origine... », (III), autour n. 34.

75. «Origine... », (III), autour n. 52. Compléter « Jalons... », (105), p. 359.

76. Els primitius..., p. 92, hypothèse reprise dans « Jalons... » (106), p. 360.

77. Ci-dessus p. 362. 
Il reste, enfin, une autre solution, certes des plus hypothétiques. Artaud de Forez, le meurtrier, aurait pu épouser une fille du premier lit de Pons de Gévaudan, sa victime. De cette union serait née Estefania, la future femme de Guillem II, qui aurait donné le prénom d'Artaud à son fils. Tout cela est, bien entendu, fondé sur les seuls critères, bien fragiles, de l'onomastique ${ }^{78}$.

\section{Mabilia, scur de Raimond Bérenger III († 1131), comte de Barcelone}

H. Débax a attiré notre attention sur la rubrique tardive d'un acte perdu du Liber Feudorum Maior, mentionnant le mariage entre un vicomte Pierre et Mabilia, sœur de Raimond Bérenger III $^{79}$. Est-ce la fille de Raimond Bérenger II ou bien d'Aimeric, vicomte de Narbonne, et de Mahaut de Pouille, auquel cas elle ne serait que la demi-sœur du comte de Barcelone ? La première solution n'est pas impossible, car le mariage entre Raimond Bérenger II et Mahaut a duré quatre ans : nous savons que Raimond Bérenger III est un fils posthume, mais rien n'empêche qu'une fille soit son aînée ${ }^{80}$.

Quant à Pierre, mari de Mabilia, la connaissance de la documentation des Trencavel empêche H. Débax d'en faire un vicomte de Carcassonne. Il existe un Pierre, vicomte d'Alt Urgell ou Castellbó, entre 1090-1150, marié depuis 1126 à Sibila, vicomtesse de Cerdagne, qui rédige son testament le 13 mai $1141^{81}$. Il se peut qu'il ait épousé en premières noces Mabilia.

Ces nouvelles identifications modifient-elles sensiblement nos conclusions sur les stratégies matrimoniales des comtes catalans aux $\mathrm{X}^{\mathrm{e}}$ et $\mathrm{XI}^{\mathrm{e}}$ siècles ? L'existence d'Ermengarde de Poitiers, Trudegarde de Narbonne et Guisla de Melgueil en tant que comtesses d'Empúries et de Roussillon, à la fin du $\mathrm{IX}^{\mathrm{e}}$ et au début du $\mathrm{X}^{\mathrm{e}}$ siècle, introduit quelques nuances à notre schéma sur le comariage des premiers comtes $^{82}$; du moins montre-t-elle qu'une endogamie poussée n'est pas incompatible avec quelques ouvertures matrimoniales. En outre, ces nouvelles données ajou-

78. T. STASSER, « Adélaïde d'Anjou... », autour n. 104-106.

79. Sacramentale Petri, vicecomitis, super honore Carcassonno (et Redes, et de matrimonio ineundo cum Amibilia, sorore eiusdem comitis), MiQuel, Liber..., $\mathrm{n}^{\circ} 851$ (1112).

80. Le 26 octobre 1081, Raimond Bérenger II et Mahaut donnent deux mille mancus à la cathédrale de Barcelone pour le bon déroulement d'un accouchement, qui est peut-être à l'origine de la naissance cette fille, Bofarull, Los condes..., t. II, p. 126, d'après les archives de la cathédrale de Barcelone, Liber Antiquitatum, fol. 89 (non vidi), analysé par F. Dingo, Historia de los victoriosíssimos antiguos condes de Barcelona, Barcelona, 1603, fol. 1.34, Raimond Bérenger III est né, treize mois plus tard, le 11 novembre 1082. Cf. en outre Dfrvic, VAISSETTE, Histoire..., t. V, n ${ }^{\circ} 6, \mathrm{col} .31$.

81. Fluvid, Els primitius... p. 160-161 et MiQuel, Liber.... n 92 (18 VI 1117 ).

82. Ci-dessus p. 364-366. 
tent Guinedilda, femme de Raimond II de Toulouse, à la génération des filles qui, autour de 930, sont mariées à des Languedociens, instaurant alors la tendance à l'exogamie dans les familles comtales ${ }^{83}$. Par ailleurs, l'abandon de l'hypothèse des origines bourguigonnes de Tota-Adelaida, devenue une Toulousaine ${ }^{84}$, place encore plus nos maisons dans la géographie du sud de la France. Leur politique matrimoniale cherche davantage l'efficacité des alliances avec les proches voisins méditerranéens que le prestige inutile du mariage lointain ${ }^{85}$. Elle les enracine de plein droit en Occitanie.

83. Ci-dessus P. 359 et Les noces..., p. 53.

84. Ci-dessus p. 368-369. Peut-être faudrait-il rejeter de même l'identification barcelonaise de Sibila (Les noces..., p. 55), si tant est que tel est son nom (cf. J. Richard, « Sur les alliances failiales des ducs de Bourgogne aux XII" et XIII' siècles », Annales de Bourgogne, 1958, p. 38), femme d'Henri de Bourgogne, et mère de Borrell, surnom que l'on retrouve ailleurs qu'à Barcelone, par exemple dans la famille seigneuriale des Courtalain, près de Châteaudun (A. Chédeville, Chartres et ses campagnes (IXe-XIII siècle), Paris, 1973, p. 320). On expliquerait mal, cependant, pourquoi le chroniqueur Rodrigo Jiménez de Rada ( $† 1247$ ) présente Henri ( +1114$)$, autre fils de «Sibila », membre de la famille ducale de Bourgogne, fondateur de la dynastie royale du Portugal, et Raimond ( $\dagger$ 1107), de la lignée comtale de Bourgogne, père d'Alphonse VII de Castille, en tant que congermani, S. de VAJAY, « Étiennette dite de Vienne, comtesse de Bourgogne : Bourgogne, Lorraine et Espagne au XIe siècle », Annales de Bourgogne, 1960, p. 239, n. 1 .

85. Cette conclusion s'inscrit dans le droit fil de l'identification d'Almodis, seconde femme de Raimond Bérenger III de Barcelone, en tant que comtesse de Melgueil, plutôt qu'avec une fille issue de la maison normande de Mortain, Les noces..., p. 391-392. Il faut corriger, sur ce point, le solide article de K.S. B. KEATS-Rohan, « The Prosopograpy of Post-conquest England : Four Case Studies », Medieval Prosopography, 1993, p. 37. 


\section{TABLEAU I : LA FAMILLE DE BELLON}

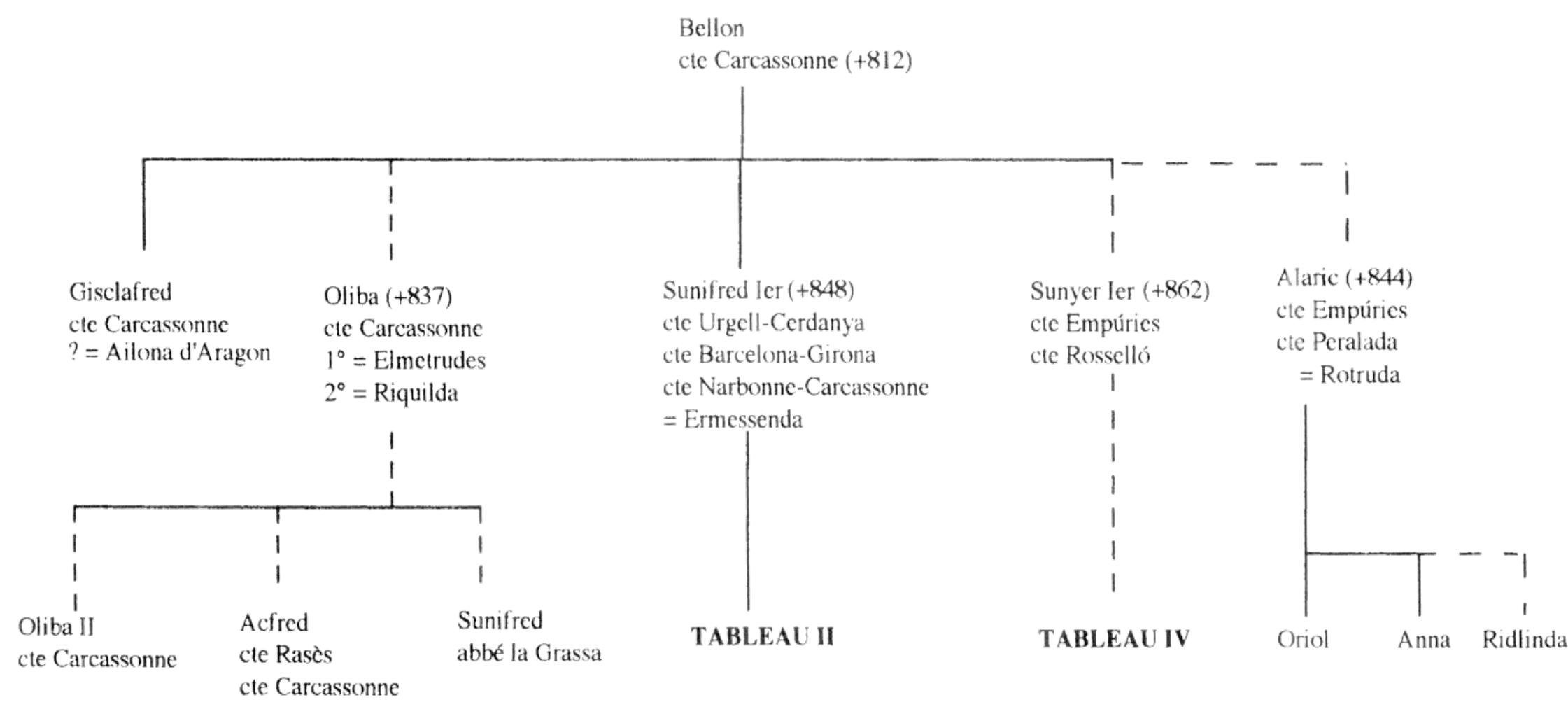




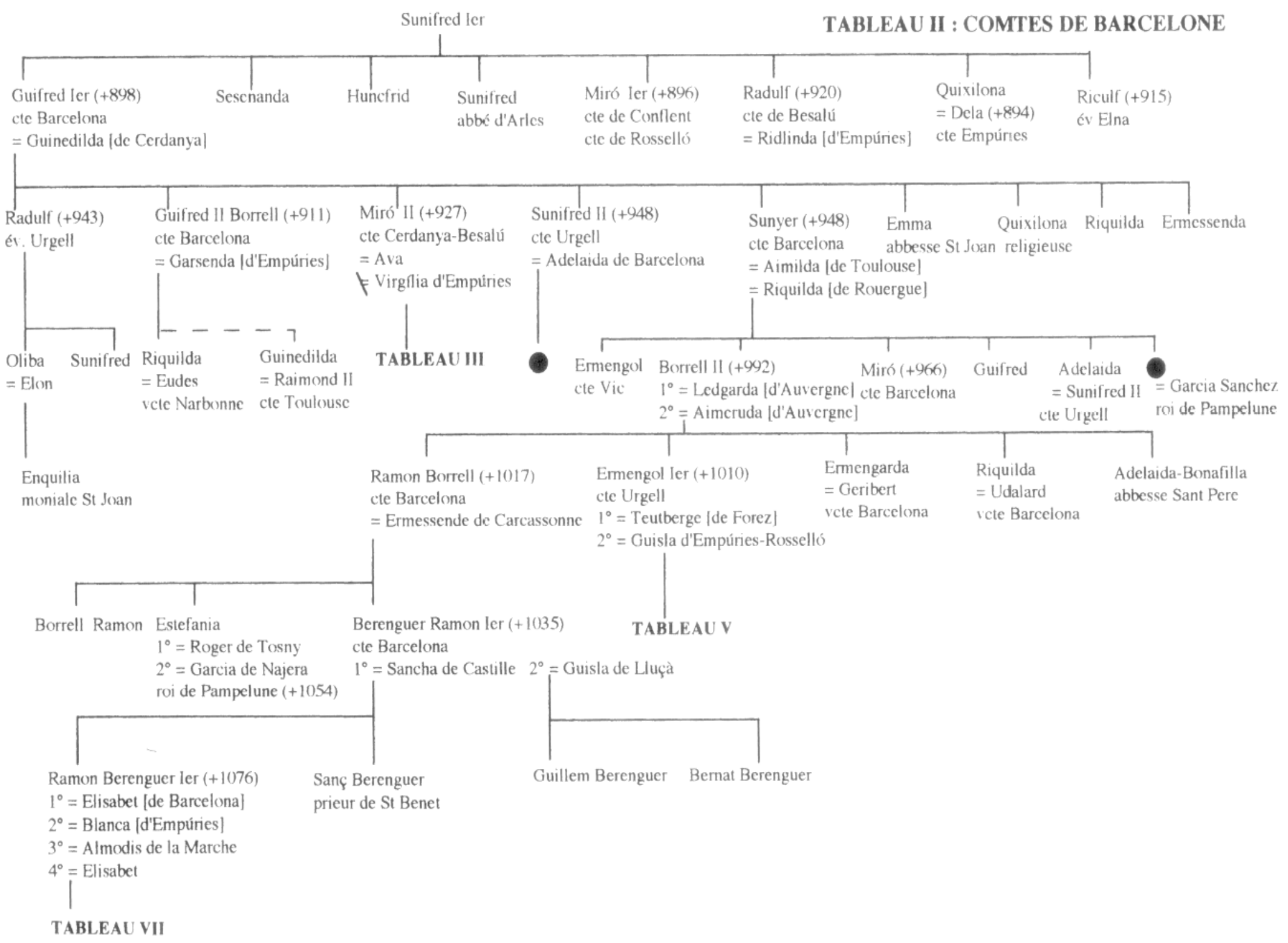




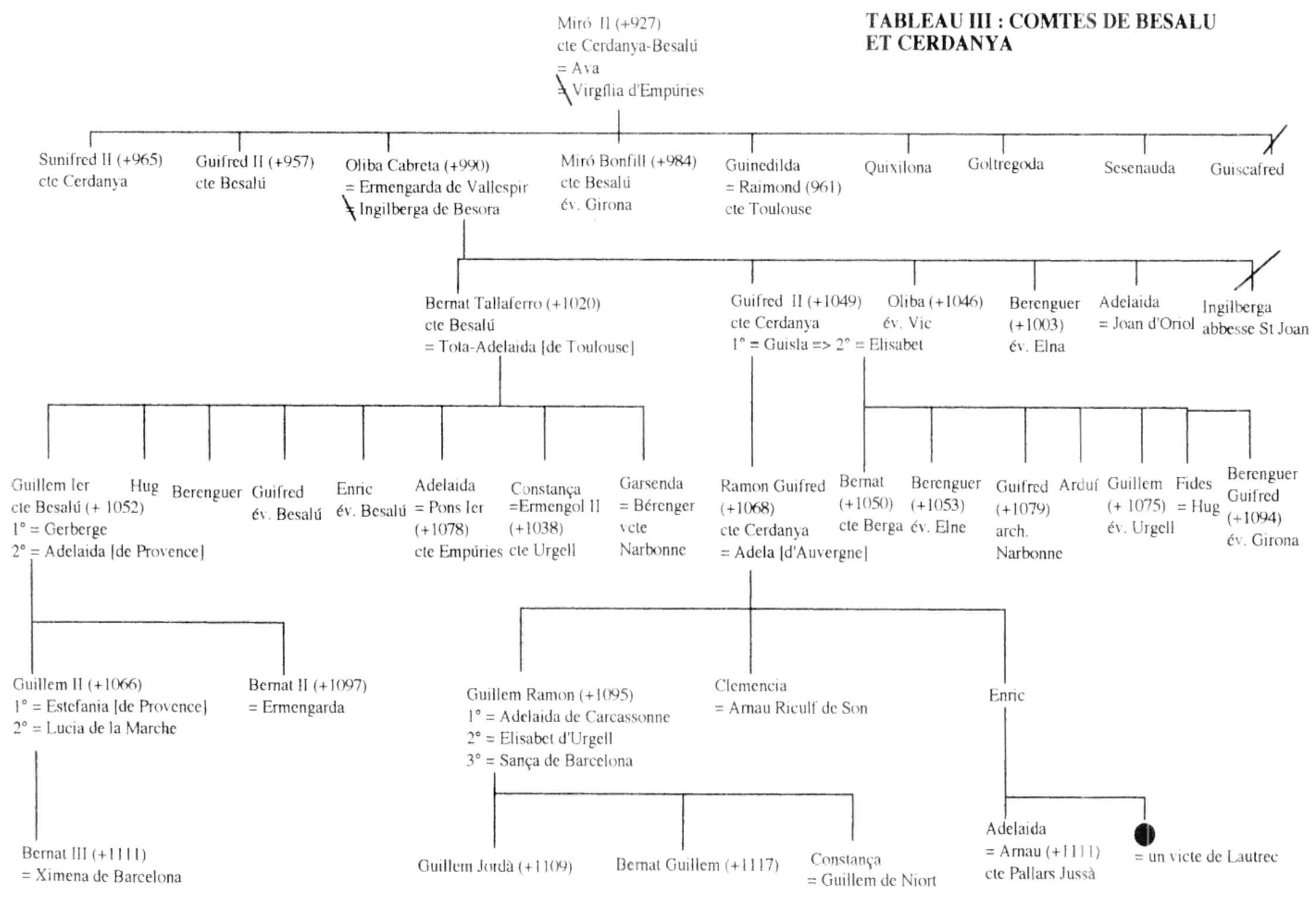




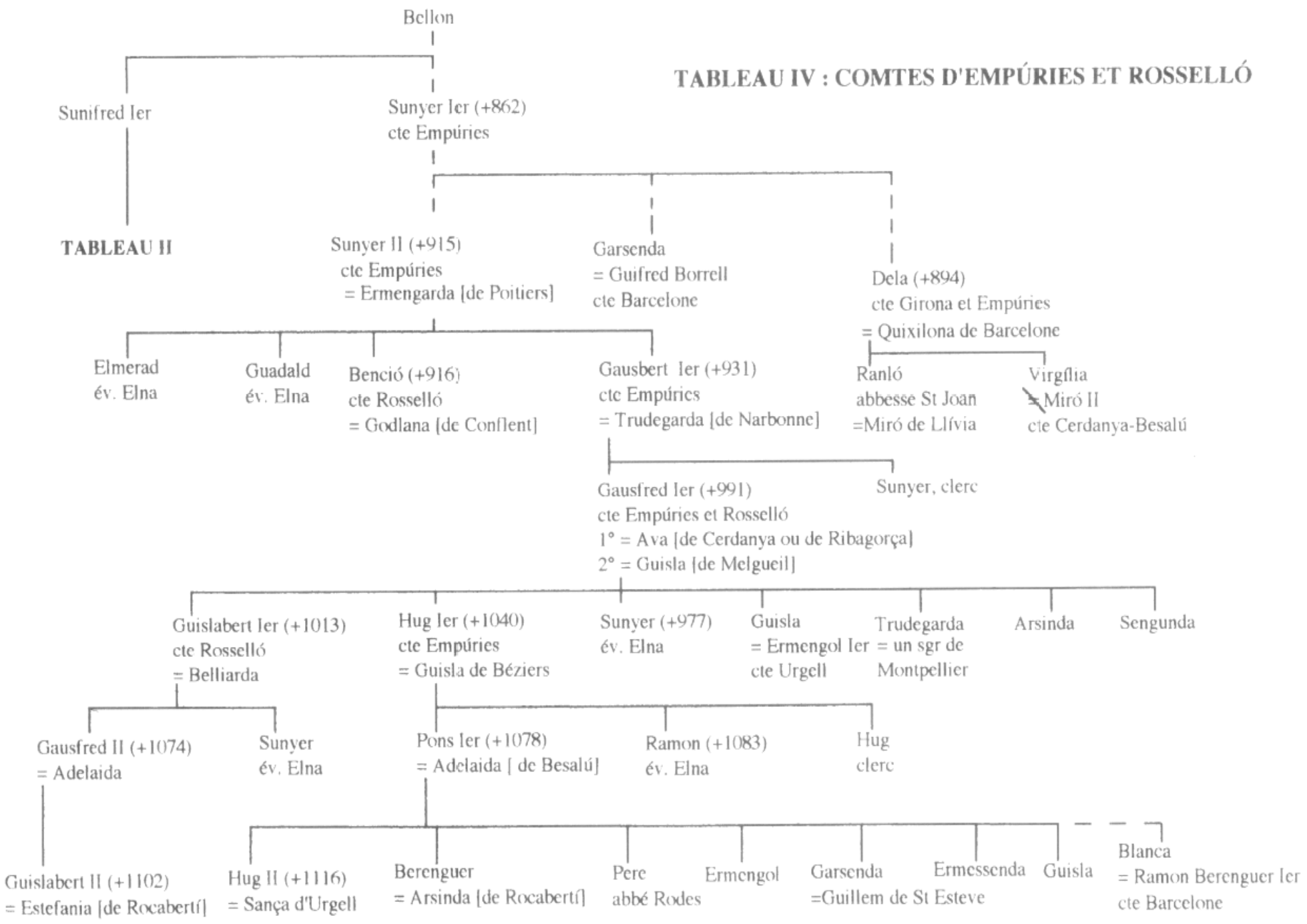


TABLEAU V : COMTES D'URGELL

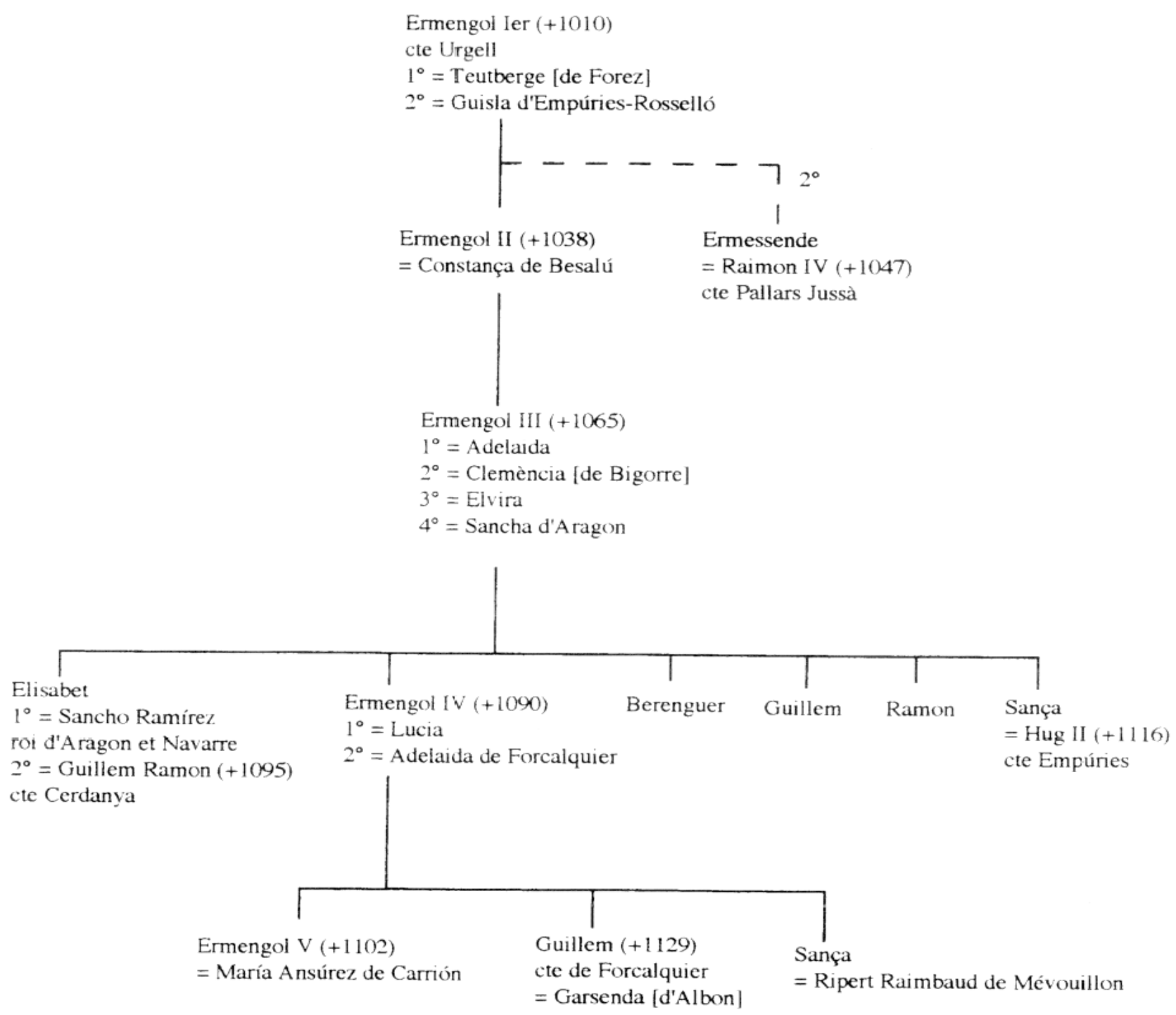


TABLEAU VI : COMTES DE PALLARS

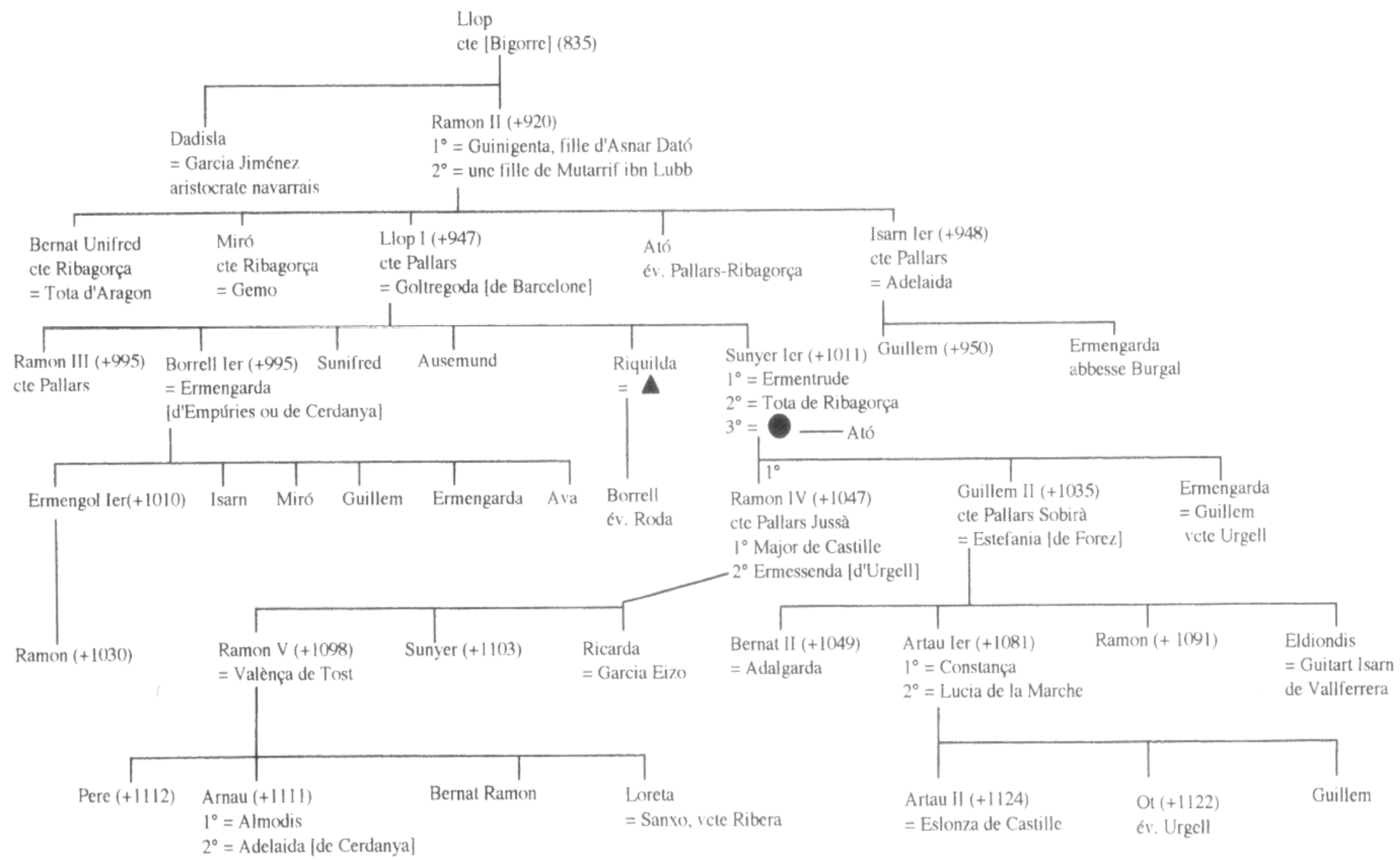




\section{TABLEAU VII : COMTES DE BARCELONE (XIIe SIECLE)}

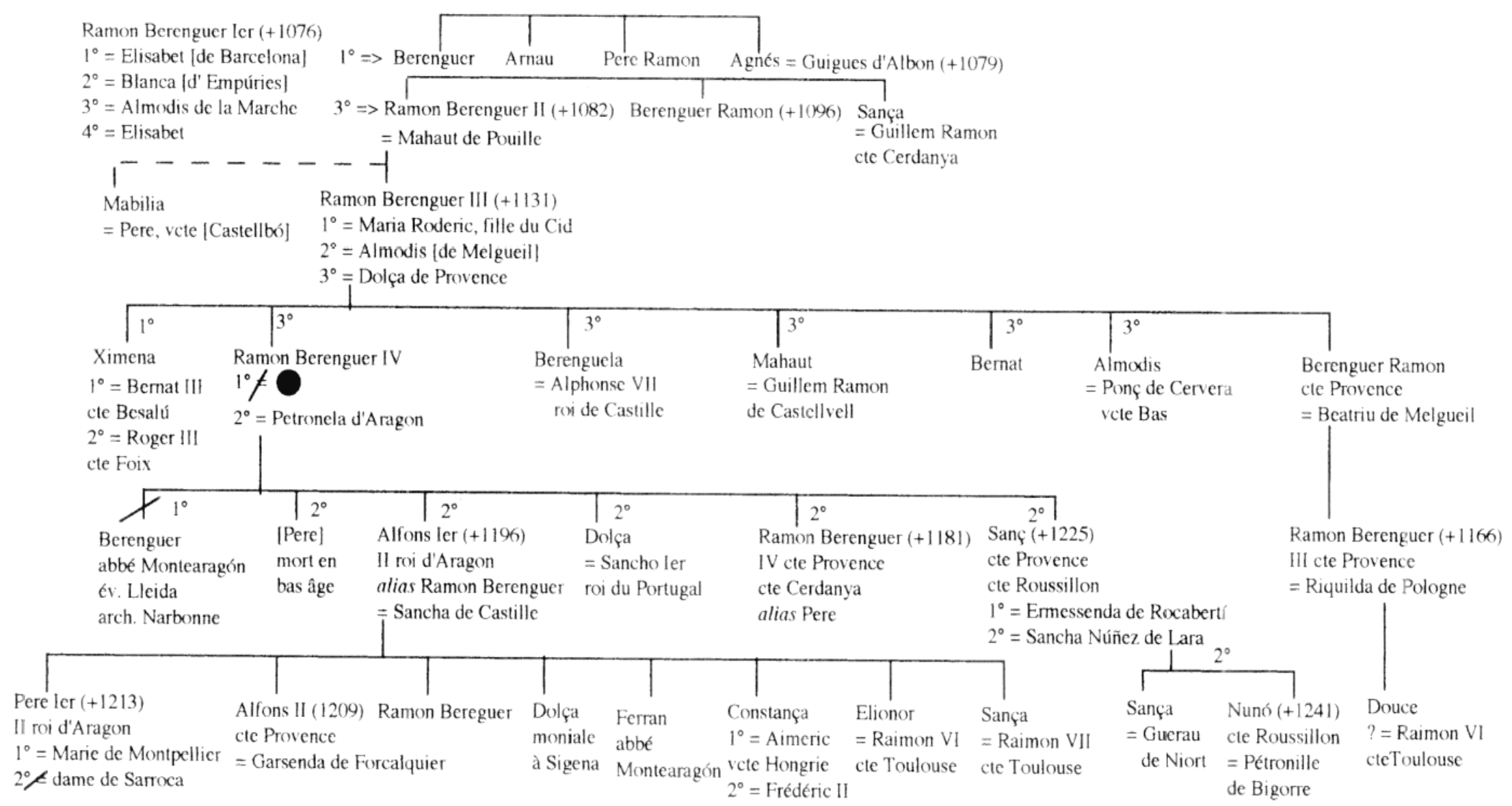

